\title{
Atomistic theory for the damping of vibrational modes in monoatomic gold chains
}

\author{
Engelund, Mads; Brandbyge, Mads; Jauho, Antti-Pekka
}

Published in:

Physical Review B Condensed Matter

Link to article, DOI:

10.1103/PhysRevB.80.045427

Publication date:

2009

Document Version

Publisher's PDF, also known as Version of record

Link back to DTU Orbit

Citation (APA):

Engelund, M., Brandbyge, M., \& Jauho, A-P. (2009). Atomistic theory for the damping of vibrational modes in monoatomic gold chains. Physical Review B Condensed Matter, 80(4), 045427.

https://doi.org/10.1103/PhysRevB.80.045427

\section{General rights}

Copyright and moral rights for the publications made accessible in the public portal are retained by the authors and/or other copyright owners and it is a condition of accessing publications that users recognise and abide by the legal requirements associated with these rights.

- Users may download and print one copy of any publication from the public portal for the purpose of private study or research.

- You may not further distribute the material or use it for any profit-making activity or commercial gain

- You may freely distribute the URL identifying the publication in the public portal

If you believe that this document breaches copyright please contact us providing details, and we will remove access to the work immediately and investigate your claim. 


\title{
Atomistic theory for the damping of vibrational modes in monoatomic gold chains
}

\author{
M. Engelund, ${ }^{1, *}$ M. Brandbyge, ${ }^{1}$ and A. P. Jauho ${ }^{1,2}$ \\ ${ }^{1}$ Department of Micro and Nanotechnology, DTU Nanotech, Technical University of Denmark, Ørsteds Plads, \\ Bldg. 345E, DK-2800 Kongens Lyngby, Denmark \\ ${ }^{2}$ Department of Applied Physics, Helsinki University of Technology, P.O. Box 1100, Helsinki FI-02015 TKK, Finland \\ (Received 17 April 2009; revised manuscript received 30 June 2009; published 30 July 2009)
}

\begin{abstract}
We develop a computational method for evaluating the damping of vibrational modes in monatomic metallic chains suspended between bulk crystals under external strain. The damping is due to the coupling between the chain and contact modes and the phonons in the bulk substrates. The geometry of the atoms forming the contact is taken into account. The dynamical matrix is computed with density-functional theory in the atomic chain and the contacts using finite atomic displacements while an empirical method is employed for the bulk substrate. As a specific example, we present results for the experimentally realized case of gold chains in two different crystallographic directions. The range of the computed damping rates confirms the estimates obtained by fits to experimental data [T. Frederiksen et al., Phys. Rev. B 75, 205413 (2007)]. Our method indicates that an order-of-magnitude variation in the harmonic damping is possible even for relatively small changes in the strain. Such detailed insight is necessary for a quantitative analysis of damping in metallic atomic chains and in explaining the rich phenomenology seen in the experiments.
\end{abstract}

DOI: 10.1103/PhysRevB.80.045427

PACS number(s): 63.22.Gh, 68.65.-k, 73.40.Jn

\section{INTRODUCTION}

The continuing shrinking of electronic devices and the concomitant great interest in molecular electronics ${ }^{1}$ have underlined the urgency of a detailed understanding of transport of electrons through molecular-scale contacts. A particularly important issue concerns the energy exchange between the charge carriers and the molecular contact. Thus, the local Joule heating resulting from the current passing through the contact and its implications to the structural stability of such contacts are presently under intense investigation. ${ }^{2-6}$ Experimentally, local heating in molecular conductors in the presence of the current has been inferred using two-level fluctuations ${ }^{7}$ and Raman spectroscopy. ${ }^{8}$

Monatomic chains of metal atoms ${ }^{9}$ are among the simplest possible atomic-scale conductors. The atomic gold chain is probably the best-studied atomic-sized conductor, and a great deal of detailed information is available from experiments $^{10-22}$ and related theoretical studies. ${ }^{13,15,18,23-33}$ The current-induced vibrational excitation and the stability of atomic metallic chains have been addressed in a few experiments. ${ }^{34-37}$

In the case of a gold chain Agraït et al. ${ }^{12}$ reported welldefined inelastic signals in the current-voltage characteristics. These signals were seen as a sharp 1\% drop of the conductance at the onset of back scattering due to vibrational excitation when the voltage equals the vibrational energy. Especially for the longer chains (six to seven atoms), the vibrational signal due to the alternating bond-length (ABL) mode 28,31 dominates. This resembles the situation of an infinite chain with a half-filled electronic band where only the zone-boundary phonon can back-scatter electrons ${ }^{11}$ due to momentum conservation.

The inelastic signal gives a direct insight into how the frequency of the ABL mode depends on the strain of the atomic chain. This frequency can also be used to infer the bond strength. The signature of heating of the vibrational mode is the nonzero slope of the conductance versus voltage beyond the onset of excitation: with no heating the curve would be flat. Fits to the experiment on gold chains using a simple model ${ }^{30}$ suggest that the damping of the excitation, as expected, can be significant. However, the experiments in general show a variety of behaviors and it is not easy to infer the extent of localization of the ABL vibration or its damping in these systems. ${ }^{38}$

In order to address the steady-state effective temperature of the biased atomic gold chain theoretically, it is necessary to consider the various damping mechanisms affecting the localized vibrations, such as their coupling to the vibrations in the contact or to the phonons in the surrounding bulk reservoirs. This is the purpose of the present paper: we calculate the vibrational modes in atomic gold chains and their coupling and the resulting damping due to the phonon system in the leads. We work within the harmonic approximation and employ first-principles density-functional theory (DFT) for the atomic chain and the contacts ${ }^{39}$ while a potential model is used for the force constants of the leads. ${ }^{40}$

Experimental transmission electron microscopy (TEM) studies ${ }^{20,41}$ have shown that atomic chains form in the $\langle 100\rangle$ and $\langle 111\rangle$ directions while the $\langle 110\rangle$ direction gives rise to thicker rods. ${ }^{41}$ Therefore we focus on chains between two (100) surfaces or (111) surfaces. We consider chain lengths of three to seven atoms and study the behavior of their vibrations and damping when the chains are stretched. The TEM micrographs also indicate that the chains are suspended between pyramids, so in our calculations we add the smallest possible fcc-stacked pyramid to link the chain to the given surfaces.

As we shall show below, at low strain the gold chains have harmonically undamped ABL modes with frequencies outside the bulk band. The long chains of six to seven atoms also have ABL modes with very low damping at high strain. Our results indicate that chains between (111) surfaces will have a lower damping than chains between (100) surfaces. Importantly, we find that the damping is an extremely sensi- 


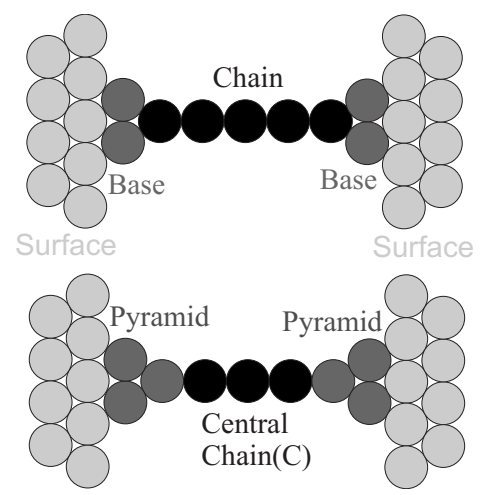

FIG. 1. Two ways of partitioning the central part of the chainsubstrate system. (Top) The chain is the part of the system that only contains one atom in a plane parallel to the surface and the base is what connects the two-dimensional surface to the chain. (Bottom) The pyramid is the base plus the chain atom closest to the base. The central chain is the remaining part of the chain after removing one atom at each end.

tive function of the external strain: an order-of-magnitude change may result from minute changes in the strain. This may provide a key for understanding the rich behavior found in experiments.

The paper is organized as follows. In Sec. II we describe how the central quantities, i.e., the dynamical matrix, the projected density of states, and the damping rates are calculated. Section III is devoted to the analysis of the numerical results we have obtained, beginning with results for the structure of the chains, proceeding to the dynamical matrix, and concluding with an analysis of the damping of modes in the systems. Section IV gives our final conclusions while certain technical details are presented in three appendices.

\section{METHOD}

As will become evident in the forthcoming discussion it is advantageous to use two different ways to label the atoms forming the junction; these two schemes are illustrated in Fig. 1. The first scheme (Fig. 1, top panel) is based on the cross-sectional area and collects all atoms with equilibrium positions on the one-dimensional line joining the two surfaces into a "chain," and calls the remaining atoms between the chain and the substrate the "base." The second scheme (Fig. 1, bottom) distinguishes between a "pyramid" and a "central chain;" this is chosen because the last atom of the chain has bonds to four or five atoms making this atom very different from the central chain atoms that only have two bonds per atom.

A quantity of central importance to all our analysis is the mass-scaled dynamical matrix, $\mathbf{K}$, which we here define as including $\hbar$,

$$
\mathbf{K}_{i j}=\frac{\hbar^{2}}{\sqrt{m_{i} m_{j}}} \frac{\partial^{2} E}{\partial u_{i} \partial u_{j}},
$$

where $E$ is the total energy of the system, $u_{i}$ is the coordinate corresponding to the $i$ th degree of translational freedom for the atoms of the system. $m_{i}$ is the mass of the atom that the

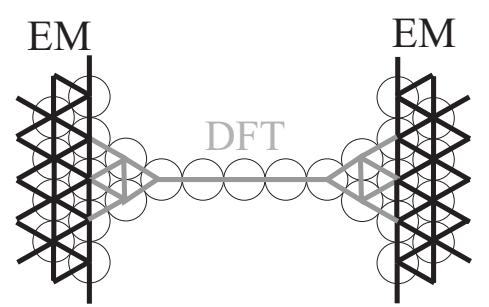

FIG. 2. Parameters used for calculating the dynamical matrix. Only nearest-neighbor coupling is shown. The coupling elements labeled "EM" are found by the empirical model and the coupling elements labeled "DFT" by DFT. Onsite elements are determined from the coupling elements (see Appendix A) and are not shown in the figure.

$i$ th degree of freedom belongs to. $\mathbf{K}$ governs the evolution of the vibrational system within the harmonic approximation. In the Fourier domain the Newton equation of motion reads

$$
\mathbf{K} u_{\lambda}=\epsilon_{\lambda}^{2} u_{\lambda}
$$

where $\lambda$ denotes a mode of oscillation in the system and $\epsilon_{\lambda}$ is the corresponding quantization energy.

The evaluation of $\mathbf{K}$ proceeds as follows. Finite difference DFT calculations (for details of our implementation, see Appendix A) were used for the chain, the base, and the coupling between the surface and the base while for the surfaces we used an empirical model due to Tréglia and Desjonquères. ${ }^{40}$ Figure 2 illustrates the domains for the two different methods. The position of the interface between the region treated by DFT and the region treated by the empirical model is a parameter that can be varied, and the dependence on the final results of the choice of this parameter is analyzed in Appendix B 2 .

The empirical model can be used to describe the onsite and coupling elements of atoms in a crystal structure. The model uses the bulk modulus of gold to fit the variation in the force constant with distance between nearest and nextnearest neighbors. Even though the empirical model is fitted to the bulk modulus, which is a low-frequency property, it still accurately predicts the cutoff of the bulk band. The positions of the neighboring atoms can only have small deviations from perfect-crystal positions (e.g., bulk, surface, and adatoms). Note that this model is general enough to give different coupling elements between surface atoms and bulk atoms. The model also distinguishes between the coupling between surface atoms with or without extra atoms added to the surface.

The DFT calculations were done with the SIESTA code, using the Perdew-Burke-Enzerhof version of the generalized gradient approximation exchange-correlation potential with standard norm-conserving Troullier-Martins pseudopotentials. We used a single-zeta-polarized (SZP) basis set with a confining energy of $0.01 \mathrm{Ry}$. A mesh cutoff of 150 Ry was used. Relaxation was done with a force tolerance of $0.002 \mathrm{eV} / \AA$. These values were found to have converged for the same type of system by Frederiksen et al. ${ }^{31,32}$ The experimental fcc bulk lattice constant of $4.08 \AA$ was used. Only the device region was relaxed (defined in Fig. 3 ). 

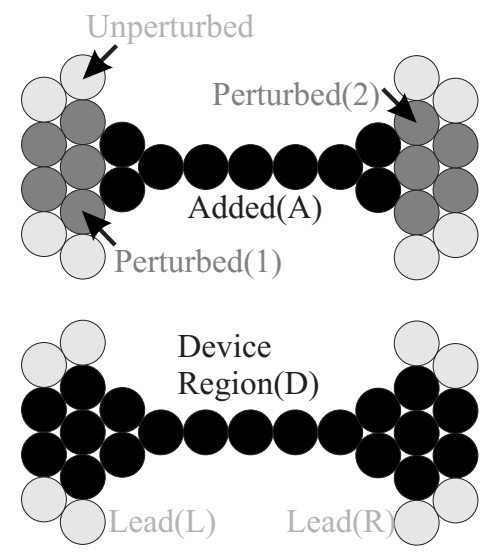

FIG. 3. Adding atoms to two surfaces. (Top) The forces between surface atoms within next-nearest-neighbor distance $4.08 \AA$ of the added atoms are perturbed by the presence of the added atoms. (Bottom) The device region is where the coupling between the atoms is different from the values for the two unperturbed surfaces. The coupling between the device region and the leads is considered to be unperturbed.

For the (111) orientations a $4 \times 4$ atom surface unit and a $2 \times 3 k$-point sampling was used while for the (100) orientations a $3 \times 3$ atom surface unit cell and a $3 \times 3 k$-point sampling was employed. This ensured a similar and sufficient $k$-point density for both kinds of surfaces (see Appendix B 2).

\section{A. Green's function for a perturbation on the surface}

All properties of interest in the present context can be derived from the (retarded) Green's function D, defined by

$$
\left[(\epsilon+i \eta)^{2} \mathbf{I}-\mathbf{K}\right] \mathbf{D}(\epsilon)=\mathbf{I} \equiv \mathbf{M D},
$$

where $\eta=0^{+}$and we defined the inverse of the Green's function by $\mathbf{M}=\mathbf{D}^{-1}$. Specifically, we shall need the Green's function projected onto the region close to the Chain. Our procedure is based on a method due to Mingo et al. ${ }^{42}$ which has previously been tested in an investigation of finite Si nanowires between $\mathrm{Si}$ surfaces. We define $\mathbf{X}_{Y Z}$ as the block of the matrix $\mathbf{X}$, where the indices run over the degrees of freedom in regions $Y, Z$, respectively, where $Y, Z=\{1,2, A, D, L, R\}$, as defined either in Fig. 1 or Fig. 3.

First, let us start with two perfect surfaces. We then add the atoms that connect these surfaces (the base and the chain). Within a certain range from the added atoms the onsite and coupling elements of $\mathbf{K}$ will be different from the values for the perfect surface. Together, the added atoms and the perturbed atoms define the device region $D$ (Fig. 3, bottom). The coupling between the device region and the rest of the surface ( $L, R$ for the left and right leads, respectively) is assumed to be unperturbed.

In order to compute the Green's function projected on the device region, $\mathbf{D}_{D D}(\epsilon)$, we first consider this matrix representation of Eq. (3): ${ }^{43}$

$$
\left(\begin{array}{ll}
\mathbf{M}_{D D} & \mathbf{M}_{D \alpha} \\
\mathbf{M}_{\alpha D} & \mathbf{M}_{\alpha \alpha}
\end{array}\right)\left(\begin{array}{ll}
\mathbf{D}_{D D} & \mathbf{D}_{D \alpha} \\
\mathbf{D}_{\alpha D} & \mathbf{D}_{\alpha \alpha}
\end{array}\right)=\left(\begin{array}{ll}
\mathbf{I}_{D D} & 0_{D \alpha} \\
0_{\alpha D} & \mathbf{I}_{\alpha \alpha}
\end{array}\right) .
$$

Here the index $\alpha=(L, R)$, i.e., the left and right unperturbed surface while $D=\{1, A, 2\}$. Using straightforward matrix manipulations one finds

$$
\mathbf{D}_{D D}=\left[\mathbf{M}_{D D}-\mathbf{M}_{D \alpha}\left(\mathbf{M}_{\alpha \alpha}\right)^{-1} \mathbf{M}_{\alpha D}\right]^{-1}=\left[\mathbf{M}_{D D}-\mathbf{\Pi}_{D D}\right]^{-1},
$$

which defines the self-energy $\boldsymbol{\Pi}_{D D}=\mathbf{M}_{D \alpha}\left(\mathbf{M}_{\alpha \alpha}\right)^{-1} \mathbf{M}_{\alpha D}$. Since the added atoms do not couple to the unperturbed surfaces, and the perturbed region 1 couples only to the right unperturbed surface while the perturbed region 2 only couples to the left unperturbed surface, the self-energy $\boldsymbol{\Pi}_{D D}$ has the matrix structure

$$
\boldsymbol{\Pi}_{D D}=\left(\begin{array}{ccc}
\mathbf{M}_{1 L}\left(\mathbf{M}_{L L}\right)^{-1} \mathbf{M}_{L 1} & 0 & 0 \\
0 & 0 & 0 \\
0 & 0 & \mathbf{M}_{2 R}\left(\mathbf{M}_{R R}\right)^{-1} \mathbf{M}_{R 2}
\end{array}\right) .
$$

This object can be evaluated as follows. First, in the limit of large regions 1 and 2, the coupling elements $\mathbf{M}_{L 1}$ and $\mathbf{M}_{R 2}$ must approach those of the unperturbed surface, $\mathbf{M}_{L 1}^{S}$ and $\mathbf{M}_{R 2}^{S}$, respectively. In what follows, we shall make the approximation that the regions 1 and 2 are chosen so that this condition is satisfied sufficiently accurately. Second, we note that the matrix $\mathbf{M}_{\alpha \alpha}$ is indistinguishable from the matrix $\mathbf{M}_{\alpha \alpha}^{S}$ as long as the involved atoms are outside the perturbed regions 1 or 2 . Therefore, we can write

$$
\begin{aligned}
& \mathbf{M}_{1 L}\left(\mathbf{M}_{L L}\right)^{-1} \mathbf{M}_{L 1} \simeq \mathbf{M}_{1 L}^{S}\left(\mathbf{M}_{L L}^{S}\right)^{-1} \mathbf{M}_{L 1}^{S} \equiv \mathbf{\Pi}_{11}^{S}, \\
& \mathbf{M}_{2 R}\left(\mathbf{M}_{R R}\right)^{-1} \mathbf{M}_{R 2} \simeq \mathbf{M}_{2 R}^{S}\left(\mathbf{M}_{R R}^{S}\right)^{-1} \mathbf{M}_{R 2}^{S} \equiv \mathbf{\Pi}_{22}^{S},
\end{aligned}
$$

where the accuracy increases with increasing size of regions 1 and 2. On the other hand, using the definition of the selfenergy, we can write

$$
\begin{aligned}
& \boldsymbol{\Pi}_{11}^{S}=\mathbf{M}_{11}^{S}-\left(\mathbf{D}_{11}^{S}\right)^{-1}, \\
& \mathbf{\Pi}_{22}^{S}=\mathbf{M}_{22}^{S}-\left(\mathbf{D}_{22}^{S}\right)^{-1},
\end{aligned}
$$

where $\mathbf{D}_{i i}^{S}, i=1,2$ is the projection of the unperturbed Green's functions onto the atoms in regions 1 and 2, respectively. This object is evaluated by exploiting the periodicity in the ideal surface plane. The Fourier transform of $\mathbf{M}^{S}$ in the parallel directions has a tridiagonal block structure and we can solve for its inverse very effectively using recursive techniques (see, e.g., Sancho et al. ${ }^{44}$ ). Of course we still have to evaluate the Fourier transform for a large number of $k$ points. The density of $k$ points as well as the size of the infinitesimal $\eta$ are convergence parameters which determine the accuracy and cost of the computation. An analysis of the choice of these parameters is given in Appendix B 2.

To sum up, the calculation is preformed in the following steps: (i) start with perfect leads and specify the device in between them. (ii) The atoms in the leads where $\mathbf{K}$ is perturbed by the presence of the device are identified. (iii) The unperturbed surface Green's function $\mathbf{D}^{S}$ is found via $k$-point sampling and then used to construct the self-energy, Eqs. (7) 
and (8). (iv) The perturbed Green's function is then found using this self-energy via Eqs. (5) and (6).

\section{B. Modes and lifetimes}

For any finite system the eigenvalues $\epsilon_{\lambda}^{2}$ and thereby also the density of states are found straightforwardly. For infinite systems we use that each eigenvector, $u_{\lambda}$, with the corresponding eigenvalue, $\epsilon_{\lambda}$ gives a contribution to the imaginary part of the Green's function in the $\epsilon_{\lambda} \gg \eta$ limit

$$
u_{\lambda}^{\dagger} \operatorname{Im} \mathbf{D}(\epsilon) u_{\lambda} \approx-\frac{1}{2 \epsilon_{\lambda}} \frac{1}{\left(\epsilon-\epsilon_{\lambda}\right)^{2}+\eta^{2}} .
$$

This expression results in the following density of states

$$
\mathbf{n}(\epsilon)=-\frac{2 \epsilon}{\pi} \lim _{\eta \rightarrow 0^{+}} \operatorname{Im} \mathbf{D}(\epsilon) .
$$

The broadened vibrational modes of the device region can each be associated with a finite lifetime. To do this we need to have a definition of an approximate vibrational mode of the central part of the system that evolves into an eigenmode of $\mathbf{K}$ when the coupling to the leads tends to zero. We define "modes" as the vectors that for some energy, $\epsilon^{*}$, correspond to a zero eigenvalue mode of $\operatorname{Re} \mathbf{D}_{D D}\left(\epsilon^{*}\right)$ (see Appendix $\mathrm{C}$ for details).

We also need to define a few characteristics of a mode. The Green's function projected onto a mode can be approximated by a broadened free phonon propagator with constants $\epsilon_{\lambda}$ and $\gamma_{\lambda}$ in a neighborhood of the mode peak energy,

$$
u_{\lambda}^{\dagger} \mathbf{D}_{D D}(\epsilon) u_{\lambda}=\frac{1}{\left(\epsilon+i \gamma_{\lambda}\right)^{2}-\epsilon_{\lambda}^{2}}=\frac{1}{\epsilon^{2}-\left(\epsilon_{\lambda}^{2}+\gamma_{\lambda}^{2}\right)+i 2 \epsilon \gamma_{\lambda}} .
$$

The time-dependent version of the Green's function is an exponentially damped sinusoidal oscillation with damping rate of $\frac{\gamma_{\lambda}}{\hbar}$, mean lifetime, $\tau_{\lambda}=\frac{\hbar}{\gamma_{\lambda}}$, and $Q$ factor, $Q_{\lambda}=\frac{\epsilon_{\lambda}}{2 \gamma_{\lambda}}$. Comparing the broadened phonon propagator to Eq. (5) we see that $u_{\lambda}^{\dagger} \operatorname{Im} \Pi(\epsilon) u_{\lambda}=-2 \epsilon \gamma_{\lambda}$, leading to

$$
\gamma_{\lambda}=-\frac{u_{\lambda}^{\dagger} \operatorname{Im} \Pi\left(\epsilon^{*}\right) u_{\lambda}}{2 \epsilon^{*}},
$$

where $\epsilon^{*}$ is the mode peak energy.

This calculation of $\gamma_{\lambda}, Q_{\lambda}$, and $\tau_{\lambda}$ only strictly makes sense for peaks with a Lorentzian line shape. This requires that $u_{\lambda}^{\dagger} \operatorname{Im} \mathbf{D}_{D D}(\epsilon) u_{\lambda}$ is approximately constant across the peak which is the case for modes with small broadening and large lifetime. Nevertheless, we will also use these definitions for the delocalized modes since the calculated values are still a measure of interaction with the leads.

We also define a measure of spatial localization, $s_{\lambda}$,

$$
s_{\lambda}=\frac{\sum_{x \in C}\left|\left(u_{\lambda}\right)_{x}\right|^{2}}{\sum_{x \in D \backslash C}\left|\left(u_{\lambda}\right)_{x}\right|^{2}} \frac{N_{D}-N_{C}}{N_{C}},
$$

where $N_{D}$ and $N_{C}$ are the number of atoms in the device and central chain region, respectively, and $D \backslash C$ means device region except the central chain (the perturbed region). This quantity is useful to pick out modes with a large amplitude in the central chain region only. We have that $s_{\lambda}=1$ signifies equal amplitude in $C$ and connecting atoms while the limit $s_{\lambda} \rightarrow \infty\left(s_{\lambda} \rightarrow 0\right)$ signifies a mode which is completely residing inside (outside) the chain.

It should be stressed that the mode properties calculated in this way only refer to the harmonic damping by the leads and that other sources of damping are not included such as electron-hole pair creation and anharmonicity. The damping due to electron-hole pair creation, obtained by an $a b$ initio calculation on a selection of gold chains, is about 50-80 $\mu \mathrm{eV}$ for the vibrational mode with the strongest coupling to electrons. ${ }^{30,31}$ This type of damping is less dependent on strain in gold chains due to stable electronic structure as evidenced by the robust electronic conductance of one conductance quantum. The harmonic damping due to the leads is typically higher than this but as we shall see it can actually drop well below this value and thus be less than the electron-hole pair damping.

In case of an applied bias the high-frequency modes may be excited to a high occupation. The creation of vibrational quanta is roughly proportional to $e V-\hbar \omega_{\lambda}$ while the damping mechanisms are not expected to have a strong dependence of the bias. Therefore, as the bias is increased beyond the phonon energy threshold, the mode occupation will rise and anharmonic interactions may become increasingly important even for low temperatures. Mingo ${ }^{45}$ has studied anharmonic effects on heat conduction in a model atomic contact and more recently Wang et $a l^{46}$ has used $a b$ initio calculations to access the effect of anharmonicity on heat conduction in carbon-based systems. Anharmonic effects are, however, outside the scope of the present work.

\section{RESULTS}

\section{A. Geometrical structure and the dynamical matrix}

In this subsection we investigate the geometrical structure of the chains and the behavior of the dynamical matrix. For each type of calculation (identified by the number of atoms in the chain, the surface orientation, and the type of base) a range of calculations were set up with the two surfaces at different separations, $L_{i}(i=1,2, \ldots)$, with the separations incremented in equally spaced steps. Trial and error was used to determine suitable step sizes for the different types of calculations.

To be able to compare chains of different lengths and between different surfaces we define the average bond length, $B=\left\langle b_{j}\right\rangle$, as the average length between neighboring atoms within the chain, where $j$ runs over the number of bonds in the chain (see Fig. 4). $B$ is useful because it is closely related to the experimentally measurable force ${ }^{14}$ on the chain and can be found without interpolation. The close relationship between $B$ and the force is demonstrated in Fig. 5 where the force is calculated as the slope of a least-squares fit of

$$
\left[\left(E_{i-1}, L_{i-1}\right),\left(E_{i}, L_{i}\right),\left(E_{i+1}, L_{i+1}\right)\right],
$$

where $E$ is the total energy. We note that the force vs $B$ curves to a good approximation follows a straight line with a 


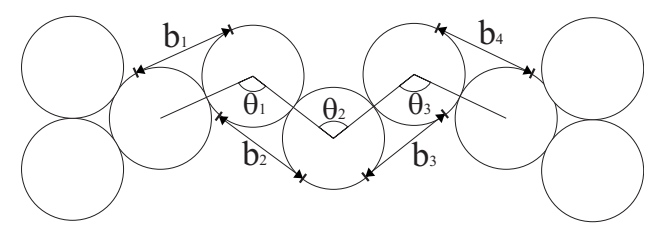

FIG. 4. Distances and angles used to define the average bond length, $B=\left\langle b_{j}\right\rangle$, and the average bond angle, $T=\left\langle\theta_{j}\right\rangle$, respectively.

slope of $k=2.5 \mathrm{eV} / \AA^{2}$, which can be interpreted as the spring constant of the bonds in the chain. In addition to $B$ we also define the average bond angle $T=\left\langle\theta_{j}\right\rangle$.

The behavior of the systems with respect to $B$ is relatively simple. As the systems are strained it is mostly the bonds in the chain that are elongated. Finally the central bond(s) become so weak that they break. At low $B$ we see from Fig. 6 that the longer chains adopt a zigzag confirmation at low average bond length. The three-atom and four-atom chains, however, remain linear within the investigated range. Furthermore, the longer chains have a similar variation in the average bond angle.

These preliminary observations are in agreement with previous theoretical studies by Frederiksen et $a .^{31}$ and Sánchez-Portal et al. ${ }^{47} \mathrm{We}$ recount these observations because we find that using $B$ as a parameter provides a helpful way to compare chain of different lengths and because the calculations in this paper are the most accurate to date. ${ }^{48}$

To shed light on the effect of straining the chains, we next investigate the energies that are related to different types of movement by analyzing the eigenmodes and eigenvalues of selected blocks of $\mathbf{K}$. Especially, we can consider the local motion of individual atoms or groups of atoms, freezing all other degrees of freedom, by picking the corresponding parts of $\mathbf{K}$. For a single atom this amounts to the onsite $3 \times 3$ blocks. The square root of the positive eigenvalues of the reduced matrix, which we call local energies, gives the approximate energy of a solution to the full $\mathbf{K}$ that has a large overlap with the corresponding eigenmode, if the coupling to the rest of the dynamical matrix is low. The negative eigenvalues of a block are ignored since they correspond to motion that is only stabilized by degrees of freedom outside the block.

The behavior of the dynamical matrix in terms of local energies is relatively straightforward, as illustrated in Fig. 7.

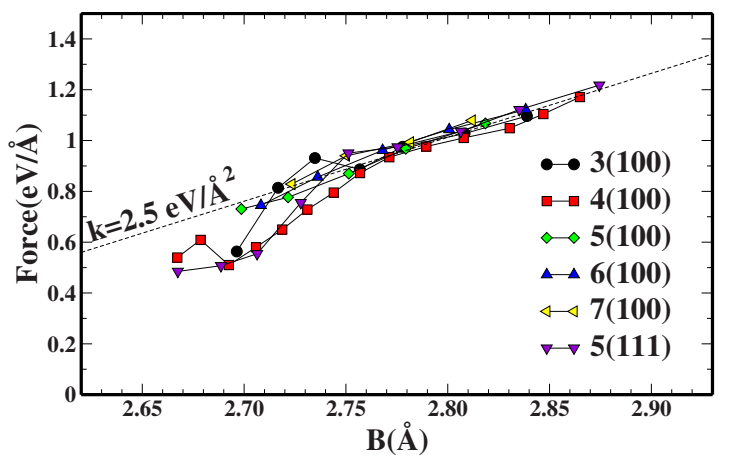

FIG. 5. (Color online) Force as a function of average bond length, $T=\left\langle\theta_{j}\right\rangle$.

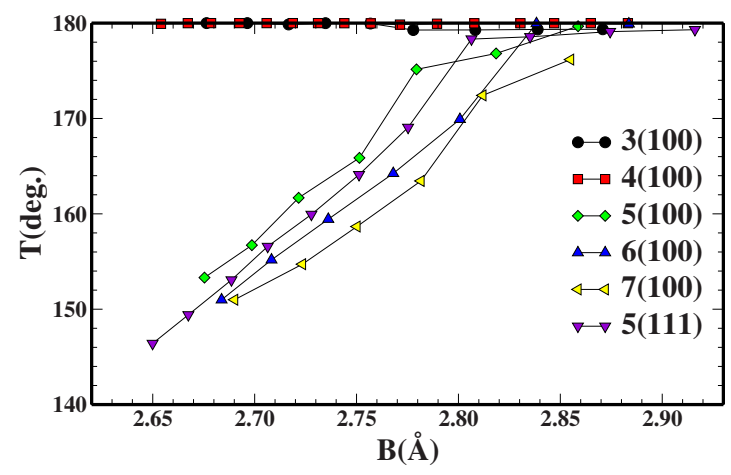

FIG. 6. (Color online) Average bond angle as a function of the average bond length. The long chains adopt a zigzag structure at low $B$ while the short chains remain linear.

When the bonds are strained they are also weakened. In the central chain the local energies are quickly reduced with increased strain $(\approx 65 \%$ decrease $)$ while the dynamical matrix of the surfaces is hardly affected. The base and the first atom of the chain fall in between these two extremes with a $20 \%$ and $40 \%$ decrease, respectively. The middle bonds in the central chain are the ones that are strained and weakened the most when the surfaces are moved apart. It is also where the chain is expected to break. ${ }^{49}$ Most interestingly, we note that at least one jump in the onsite local energies occur when moving from the surface to the central chain.

In Fig. 8 we see how motion parallel to the chain is at higher energies than perpendicular motion, and that the longitudinal optical (LO)-type motion of the ABL modes has the highest energy. We also see that the local energies of the ABL/LO-type motion moves past the local energies of the pyramid as the strain is increased. In this way the ABL/LO modes can in some sense act as a probe of the contacts.

\section{B. Mode lifetimes and $Q$ factors}

We next investigate the modes of the finite chain systems. An example is given in Fig. 9 which depicts the projected density of states (DOS) for a chain with four atoms at an intermediate strain. Notice the large variation in the width of the peaks. The peaks with a low width correspond to modes that have the largest amplitude in the chain while the peaks with a large width correspond to modes with large amplitude

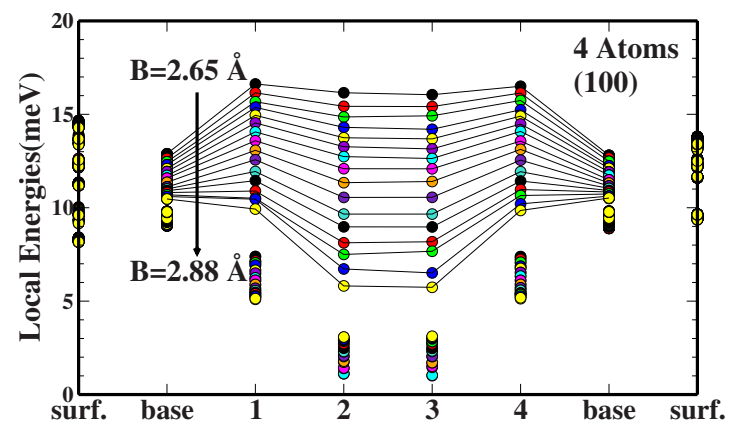

FIG. 7. (Color online) Local energies of a four-atom chain between two (100) surfaces at different strains. The largest eigenvalues are connected by a line to guide the eye. 


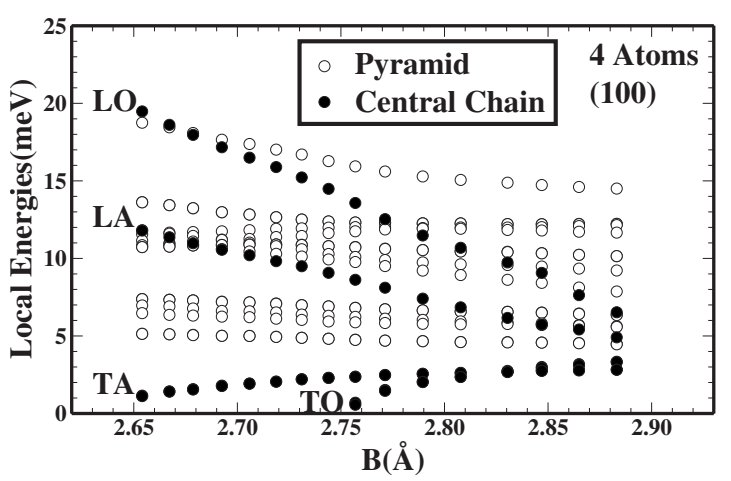

FIG. 8. Local energies for selected blocks of $\mathbf{K}$ (pyramid/central chain) plotted vs the average bond length in the four-atom chain. Since the central chain in this case consists of two atoms we can classify the eigenvectors as LO: longitudinal optical, LA: longitudinal acoustic, TO: transverse optical (doubly degenerate), or TA: transverse acoustic (doubly degenerate).

on the base and surface. Since this type of system has no natural boundary between "device" and "leads" we will have large variation in the harmonic damping no matter where we define such a boundary.

In Fig. 10 we present the $Q$ factor, spatial localization, and peak energy of all modes for chains with three to seven atoms between (100) surfaces. These are the main results of this paper. Table I shows the same information in an alternative form. We now proceed to an analysis of these results.

The ABL modes are of special interest. These modes have been identified by previous theoretical and experimental studies as the primary scatterers of electrons. ${ }^{12,28-30,32,33,50}$ The ABL modes are easily identified in Fig. 10 since they have the highest energy of the modes that are spatially localized to the central chain (black or dark gray in the figure). Modes corresponding to transverse motion of the central chain are also clearly visible. These modes are energetically and spatially localized but are of limited interest because of a low electron-phonon coupling.

Certain ABL modes are very long lived. At low strains, ABL modes lie outside the bulk (and surface) band and have, in our harmonic approximation, an infinite $Q$ factor. In reality the $Q$ factor will be limited by electron-phonon and anharmonic interactions. At higher strain the ABL modes move inside the bulk band and one observes a great variation in the

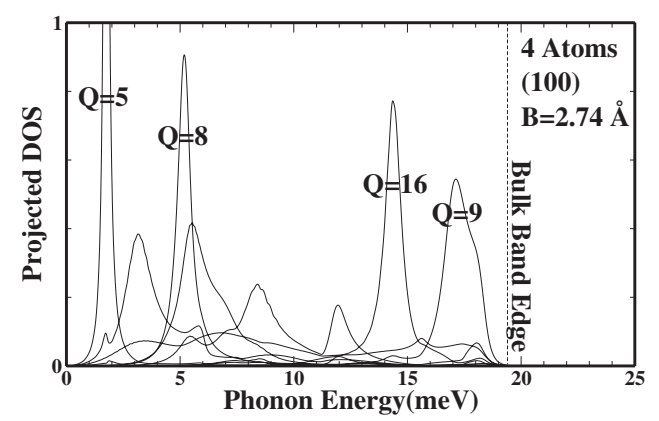

FIG. 9. Projected DOS onto a representative selection of the vibrational modes of the device region that has a large overlap with the added (chain+base) region. corresponding $Q$ factors. When the peak energy lies inside the bulk band there exists modes in the bulk with the same energy and it will mostly be the structure of the connection between the bulk crystal and the chain that determines the width of the peak.

The long chains tend to have longer lived ABL modes due to the larger ratio between the size of the central chain and the size of its boundary. The seven-atom chain is especially interesting since it has an ABL-type mode with a damping of $5 \mathrm{meV}$ at one strain while at another strain the ABL mode has a damping of $300 \mathrm{meV}$. This is more than one order-ofmagnitude variation in the harmonic damping of the primary scatterer of electrons due to only a $0.03 \AA$ change in the average bond length.

The largest damping of an ABL mode for these systems is $\gamma_{\lambda} \approx 1 \mathrm{meV}$, which is still significantly lower than the $\approx 20 \mathrm{meV}$ bandwidth. This can be attributed to fact, noted above, that there always exists a large mismatch in local energies moving from the central part of the chain to the rest of the system (see Fig. 7).

Previous studies by Frederiksen et al. ${ }^{31}$ obtained a rough estimate for the variation in the nonelectronic (harmonic and anharmonic) damping of 5-50 $\mu \mathrm{eV}$ for the longer chains by fitting the experimental inelastic electron tunneling spectroscopy (IETS) signals of Agraït et al. ${ }^{11}$ to a model calculation. The estimated peak energies lie well within the bulk band for all the recorded signals. The reason the nonelectronic damping rate can be extracted is because the excitation of vibrations and damping of vibrations through electron-hole creation are both proportional to the strength of the electronphonon coupling. This means that the step in the experimental conductance, when the bias reaches the phonon energy, can be used to estimate strength of the electronphonon interaction and thereby the electron-hole pair damping. The slope in the conductance beyond this step can then be used to extract the total damping. By subtracting the electron-hole pair damping from the total damping we get an estimate of the sum harmonic and anharmonic contributions to the damping.

The estimate in Ref. 31 agrees well with our lowest damping of $5 \mu \mathrm{eV}$. The highest damping we have found was $\approx 400 \mu \mathrm{eV}$ found for the six-atom chain which is an order of magnitude larger than the upper limit of Ref. 31. We believe that this discrepancy can be largely attributed to the difficulty in extracting the necessary parameters from experiments when the harmonic damping is large. Furthermore, for the six-atom and seven-atom chains we observe that the high damping occurs at low strain, where the electron-phonon coupling is weak. ${ }^{11}$

There are two main differences between the (100) and the (111) systems. The first difference is that the (111) systems have ABL modes that are more long lived compared to the (100) systems (see Table I and Fig. 11). The second difference is the behavior of the localized modes close to the band edge (see Fig. 11). The modes with energies outside the bulk band in the (111) systems are less spatially localized compared to the (100) case. At low strain, the (111) chain has $\mathrm{ABL}$ modes extending further into the base and surface than the (100) chain.

There are certain general features of how the damping evolves with strain that are easily understood. Modes with 


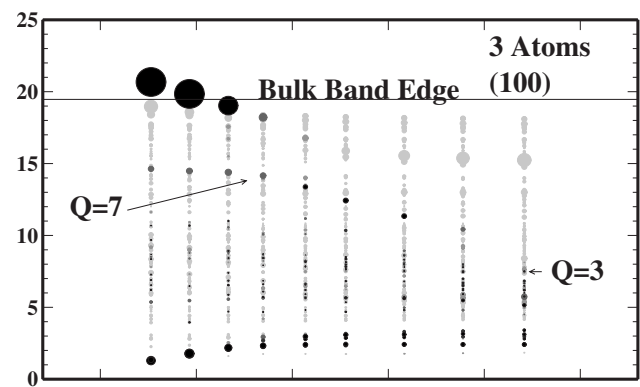

(a)

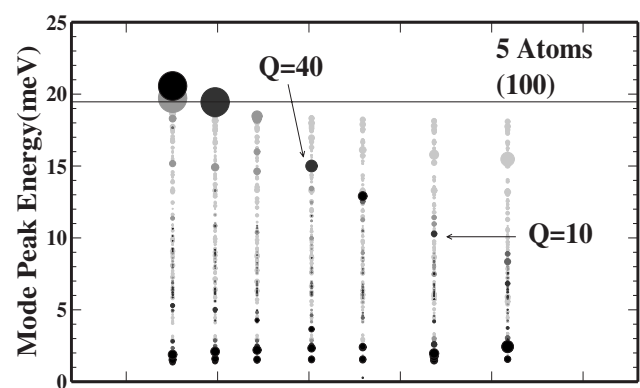

(c)

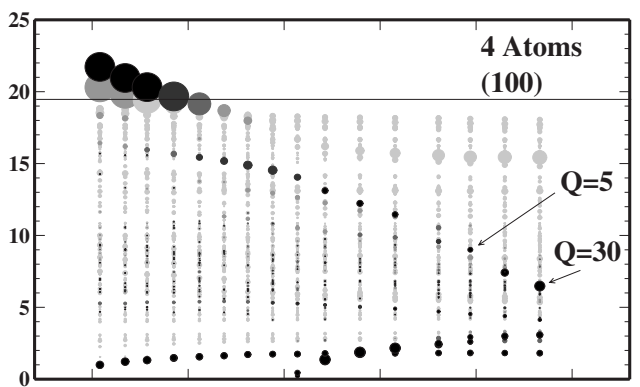

(b)

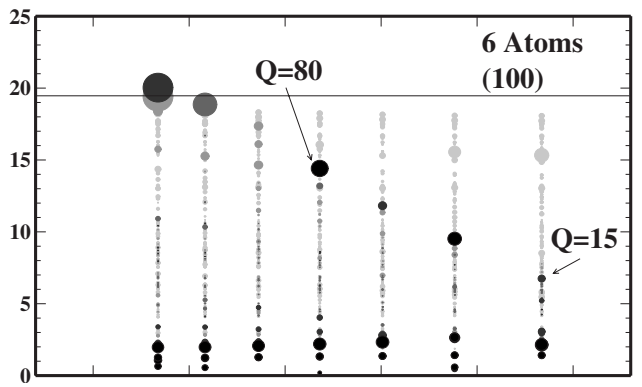

(d)

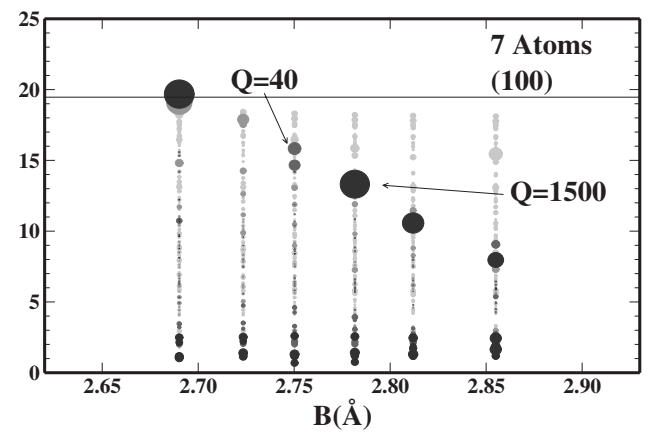

(e)

FIG. 10. The vibrational modes for chains with three to seven atoms between two 100 surfaces. The center of the disks is positioned at the peak of the projection of vibrational DOS on the mode in question. The area of a disk is proportional to the $Q_{\lambda}$ but is limited to what corresponds to a $Q$ factor of 250. The gray level that ranges from light gray to black in four steps signifies that $s_{\lambda} \in$ [0,2[ (light gray), $[2,4[,[4,6[,[6,8[$, or $[8, \infty[$ (black).

peak energies in the range of $16-19 \mathrm{meV}$ in general have a very high damping while those in the range of $14-16 \mathrm{meV}$ have very low damping. This correlates well with the bulk DOS for gold (see, e.g., Ref. 40). The optical peak in the bulk DOS corresponds to strong damping while the gap between optical and acoustical modes correspond the range of low damping.

To sum up, localized modes occur at low strain where the bonds in the chain are very strong and give rise to frequencies close to or outside the bulk band edge. Inside the bulk band strong localization is still possible for the long chains, especially the seven-atom chain. This requires, however, that the coupling between the central chain and the surface is weak at the typical frequency of the ABL mode due to the structure of the connection. The behavior depends strongly on the detailed structure of the base and the state of strain but some general features can be related to the bulk DOS.

\section{CONCLUSION AND DISCUSSION}

We have presented a study of the harmonic damping of vibrational modes in gold chains using a method that uses $a b$ initio parameters for the chains and empirical parameters for the leads. We have focused on ABL/LO modes that interact strongly with electrons and are thereby experimentally accessible through $I V$ spectroscopy. We provide an estimate for the damping of ABL modes from $a b$ initio calculations as a function of strain for a wide range of gold chain systems. The calculations of the ABL-phonon damping rates agree well with earlier estimates, found by fitting a model to experimental inelastic signals. ${ }^{11,32}$

We have found that the values of the harmonic damping for the ABL modes can vary by over an order of magnitude with strain. Even with small variations in the strain, the harmonic damping can exhibit this strong variation. This extreme sensitivity may explain the large variations seen experimentally in different chains. 
TABLE I. The variation in the $Q_{\lambda}, \gamma_{\lambda}$, and $\tau_{\lambda}$ of the ABL modes. For chains with 3-7 between (100) surfaces and for chains with five atoms between (111) surfaces but with slightly different bases. The strains where the peak energy of the ABL mode falls close to or outside the bulk band edge have been disregarded.

\begin{tabular}{cccc}
\hline \hline Chain & $Q_{\lambda}$ & $\begin{array}{c}\gamma_{\lambda} \\
(\mu \mathrm{eV})\end{array}$ & $\begin{array}{c}\tau_{\lambda} \\
(\mathrm{ps})\end{array}$ \\
\hline $3(100)$ & $3-7$ & $800-1200$ & $0.5-0.8$ \\
$4(100)$ & $5-30$ & $100-900$ & $0.7-7$ \\
$5(100)$ & $10-40$ & $200-500$ & $1.3-3$ \\
$6(100)$ & $15-80$ & $90-400$ & $1.6-7$ \\
$7(100)$ & $40-1500$ & $5-300$ & $2-130$ \\
$5(111)$ (symmetric) & $15-80$ & $40-400$ & $1.6-16$ \\
$5(111)$ (asymmetric) & $10-100$ & $40-800$ & $0.8-16$ \\
\hline \hline
\end{tabular}

The range of the harmonic damping also depends strongly on the number of atoms in the chain since we see a clear increase in localization going from a six-atom to a sevenatom chain. The chain with seven atoms really stands out, since it, in addition to having very localized modes in general, it also has the greatest variation in harmonic damping. This strong variation in the harmonic damping of the ABL modes, that depends on the details of the structure, suggest that accurate atomistic calculations of the vibrational structure is necessary to predict the inelastic signal.

All types of chains were found to have ABL modes that lie outside the bulk phonon band at low strain. These modes are expected to have very long lifetimes since the harmonic damping is zero. Signatures of the rather abrupt change in the damping of the ABL modes when strained have not been discussed in experimental literature so far. We believe this is due to the common experimental techniques for producing these chains heavily favor strained chains. The ABL-mode lifetime may be set by the coupling to the electronic system (electron-hole pair damping). Indeed, even inside the bulk band the electron-hole pair damping can be of the same order as the harmonic damping. For example, a $\gamma_{\lambda e h} \approx 50-80 \mu \mathrm{eV}$ was found for a four-atom ${ }^{30}$ and a seven-atom ${ }^{31}$ chains, which we can compare with $100-900 \mu \mathrm{eV}$ and 5-300 $\mu \mathrm{eV}$ found above for the harmonic vibrational damping. Thus the damping can in certain cases be dominated by the electron-hole pair damping for frequencies even inside the bulk band.

Finally we find a difference in the damping of ABL modes in chains between (100) and (111) surfaces. For the investigated five-atom chains there is both a marked difference in the strength of damping and in the variation in the damping with strain. It might be possible to distinguish between (100) and (111) pyramids experimentally due to this difference. The ABL modes will have strong coupling to the bulk at certain energies, characteristic of the pyramid type. This in turn, results in broadening/splitting of the modes depending on whether the characteristic energies are inside or outside the bulk band. This broadening/splitting would be detectable in the $I V$ curve since it is related to the characteristics of the conductance step at the peak energy of the vibrational mode. Finding $I V$ curves at different strains could thereby serve as

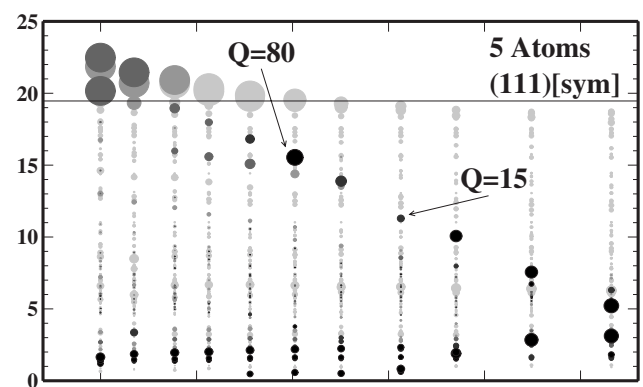

(a)

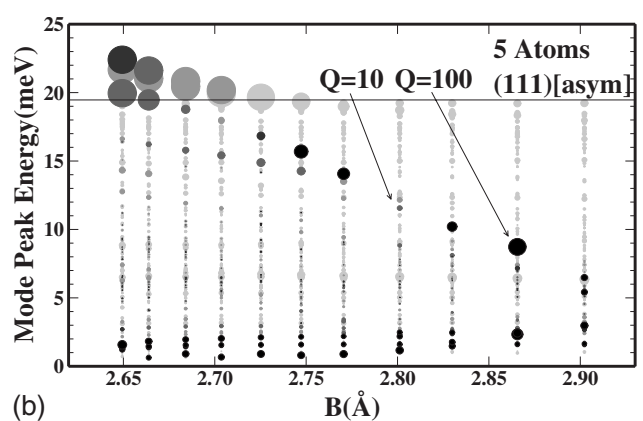

FIG. 11. The vibrational modes for five-atom chains between two (111) surfaces. (Top) Symmetric pyramids. (Bottom) Asymmetric pyramids (one atom added to one of the pyramids). The area of a disk is proportional to the $Q_{\lambda}$ but is limited to what corresponds to a $Q$ factor of 250 . The gray level that ranges from light gray to black in four steps signifies that $s_{\lambda} \in[0,2[$ (light gray), $[2,4[,[4,6[,[6,8[$, or $[8, \infty[$ (black).

a fingerprint of the specific way the chain is connected to the surroundings. Hihath et al. ${ }^{50}$ have demonstrated that such measurements are indeed possible on a single-molecule contact.

The techniques used in this paper can be combined with electronic transport calculations to predict the inelastic signal in the $I V$ characteristic of a system. This will be done in future work, where we will also eliminate the use of the empirical model for the leads and use ab initio parameters for the entire system.

\section{ACKNOWLEDGMENTS}

The authors would like to thank Thomas Frederiksen for helpful discussions and Nicolas Agraït for showing his unpublished experimental results. A.P.J. is grateful to the FiDiPro program of the Finnish Academy. Computational resources were provided by the Danish Center for Scientific Computing (DCSC).

\section{APPENDIX A: CONSTRUCTING THE DYNAMICAL MATRIX}

In this subsection the details of how we constructed the dynamical matrix are presented. The dynamical matrix must be symmetric and obey momentum conservation. Momentum conservation, in this context, means that when an atom is displaced the force on the displaced atom equals minus the total force on all other atoms. We ensure momentum conser- 
vation by setting the onsite $3 \times 3$ matrix to minus the sum of the force-constant coupling matrices to all the other atoms. This method for regularizing the dynamical matrix was previously used by Frederiksen et al. ${ }^{31}$ and generally improves on the errors introduced in the total energy when displacing atoms relative to the underlying computational grid (the DFT egg-box effect). We calculate off-diagonal coupling part of the force-constant matrix was calculated with a finite difference scheme using a displacement, $Z$, of $0.02 \AA$ in the $x, y$, and $z$ directions for all atoms in the chain and base.

To improve the accuracy further, the forces were calculated for both positive and negative displacements. If $i$ and $j$ are degrees of freedom situated inside the DFT region we therefore perform four independent calculations of $\mathbf{K}_{i j}=\mathbf{K}_{j i}$ since $\mathbf{K}$ is a symmetric matrix. In the end we use the average of the force constant from these four calculations

$$
\mathbf{K}_{i j}=\frac{\hbar^{2}}{\sqrt{m_{i} m_{j}}}\left(\frac{F_{i j+}}{Z}+\frac{F_{j i+}}{Z}-\frac{F_{i j-}}{Z}-\frac{F_{j i-}}{Z}\right) / 4,
$$

where, e.g., $F_{i j+}$ denotes the force on $i$ due to a positive displacement of $j$. If $i$ is inside the DFT region and $j$ is not, the coupling is calculated as an average of two force constants

$$
\mathbf{K}_{i j}=\frac{\hbar^{2}}{\sqrt{m_{i} m_{j}}}\left(\frac{F_{j i+}}{Z}-\frac{F_{j i-}}{Z}\right) / 2 .
$$

If an atom was close to a periodic image of another atom (less than half the unit-cell length in any direction) the force between these atoms was set to zero to avoid artifacts of the periodic calculational setup. The empirical model was used to calculate the coupling between the surface atoms. After all coupling elements were found the onsite elements were calculated for the system as a whole.

\section{APPENDIX B: CONVERGENCE}

\section{Convergence parameters}

In the calculations there are several convergence parameters and here we provide an overview. There are three important length scales in the calculations: $L_{1}, L_{2}$, and $L_{3}$. We assume that when two atoms are further apart than $L_{1}$, the coupling elements between them vanishes. $L_{2}$ is the correlation length for properties that do not have an energy dependence, such as forces, equilibrium positions, and total energies while $L_{3}$ is the assumed correlation length for properties that do have an energy dependence, such as the surface Green's function, vibrational DOS, etc. $L_{3}$ always needs to be larger than $L_{2}, L_{3}>L_{2}$ but the specific size needed depends on the required energy resolution. $L_{2}$ determines the $k$-point sampling used in the DFT calculations and $L_{3}$ the $k$-point sampling used in the calculation of the surface Green's function. In each case the number of $k$ points used one direction is chosen to be the smallest integer, $i$, such that $i>\frac{L}{a}$, where $a$ is the size of the calculational cell in that direction. The DFT $k$-point sampling used is dense enough to ensure that $L_{2}>23 \AA$ for all calculations.

In the calculation of the Green's functions we introduced a finite artificial broadening. This broadening, $\eta$, was divided into a small broadening of the device region, $\eta_{C}$, and a large broadening for the leads, $\eta_{L}$. The reasoning behind this is that the density of states is much more smooth in the bulklike regions far away from the chain. A large $\eta_{L}$ has the advantage that it reduces the need for $k$-point sampling drastically. Without a small $\eta_{C}$ we would not be able to discover very sharp peaks in the DOS. To reliably find the modes of the system it is also important that the energy spacing, $\Delta E$ is on the same level or smaller than $\eta_{C}$.

The artificial broadening limits how large lifetimes we can resolve. This is why we in the following write the upper limit to the lifetime introduced by the artificial broadening.

A final convergence parameter is the position of the interface between DFT and empirical model parameters for the dynamical matrix. This is a very important parameter since the error introduced by having this interface relatively close to the chain is what limits the precision of the calculations.

\section{Test of convergence}

Next we present the tests that have been carried out to ensure that the calculations in this paper are sufficiently converged. The convergence for the SIESTA basis set and the size of the finite displacement used in the finite difference calculations was already tested for the same type of systems by Frederiksen et al. ${ }^{31}$

So here we first examine the convergence of the DFT calculations of the dynamical matrix. A calculation for a four-atom chain between (100) surface was done with improved values for the important DFT convergence parameters. The mesh cutoff was increased from 150 to $200 \mathrm{Ry}$ and the $k$-point sampling was increased from $2 \times 3$ to $3 \times 4$. For this change in parameter we obtained a maximal difference of $0.2 \mathrm{meV}$, when comparing the square root of the sorted array of eigenvalues of the dynamical matrix. This is a negligible size since the average value of the eigenvalues is about $10 \mathrm{meV}$. The $k$-point sampling in the DFT calculations proved crucial for the structure of the strained systems since gamma-point calculations resulted in different structures (different bonds weakened at high strain) with very large lifetimes.

The perturbation length used in our calculations was $L_{1}$ $=4.08 \AA$, which is the same as next-nearest-neighbor distance. The magnitude of any next-nearest-neighbor coupling matrix, defined as $|\mathbf{X}|=\sqrt{\sum_{i j} X_{i j}^{2}}$ was never larger than $15 \%$ compared to the magnitude of any nearest-neighbors coupling matrix. The error introduced by this truncation is smaller than the one introduced by using the empirical model for the dynamical matrix.

For the calculation of the DOS we gradually improved $L_{3}$, $\eta_{L}$, and $\eta_{C}$ and found that the DOS was converged using $\Delta E=10 \mu \mathrm{eV}, \eta_{L}=100 \mu \mathrm{eV}(7 \mathrm{ps}), \eta_{C}=10 \mu \mathrm{eV}(70 \mathrm{ps})$, and $L_{2}=200 \AA(68 \times 68 k$ points $)$ except in one calculation for the seven-atom chain we needed the lifetime of one very sharp peak. This required a better resolution using $\Delta E$ $=1 \mu \mathrm{eV}, \eta_{L}=10 \mu \mathrm{eV}(70 \mathrm{ps}), \eta_{C}=1 \mu \mathrm{eV}$ (700 ps), and $L_{2}=400 \AA(136 \times 136 k$ points $)$.

Finally, we have considered how much the interface between the DFT parameters and the empirical parameters af- 


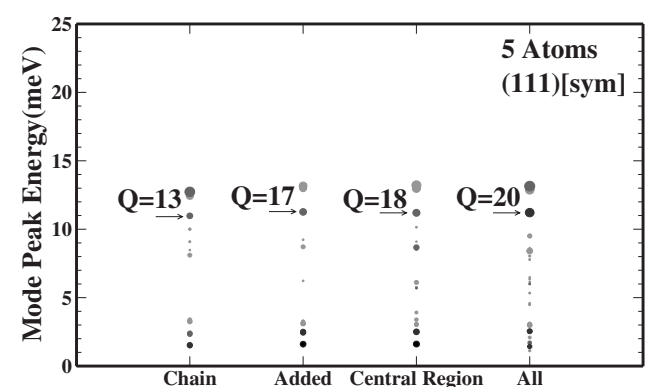

FIG. 12. Modes of the device region with a different DFT/EM interface. The label designates the region treated with DFT, where the "added" region is the one used in the main part of the calculations and "all" is fully $a b$ initio. See Fig. 11 to see what the color and size signify. In this plot modes with $s_{\lambda} \in[0,2[$ are suppressed.

fects our results. In Fig. 12 we show a study where we vary the position of this interface. We find that our calculation of the $Q$ factor and the spacial localization is converged to about the first significant digit for modes that are spatially localized to the central chain. We judge that this is what mainly sets the limit of accuracy of in our calculations.

\section{APPENDIX C: DEFINITION OF THE MODES FOR AN OPEN SYSTEM}

The starting point is the modes of a closed system, namely, the eigenmodes of $\mathbf{K}$. The most important requirement, for the definition of modes in the case of the open system, is that these modes become the modes of the isolated system in the limit of zero coupling between the device region and the leads.

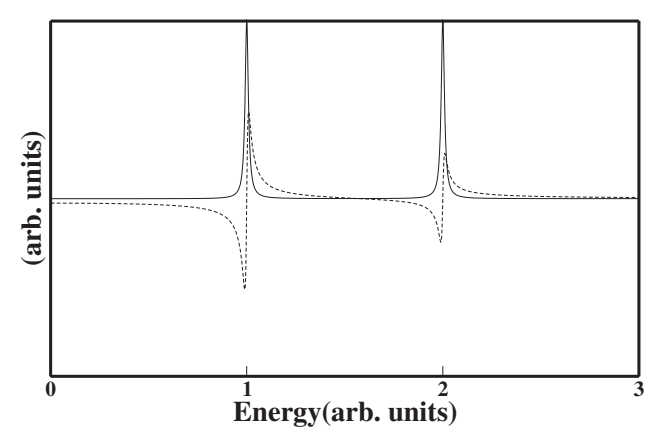

FIG. 13. Example Re D and $n(\epsilon)$, dashed and solid line, respectively, for a Green's function with two poles at 1 and 2 with a 0.1 broadening. We see that the values where the real part is zero only correspond to peaks in the density if the slope is positive.

The following definition fulfills this condition. A mode is defined as a (complex) eigenvector $u_{\lambda}$ of $\mathbf{M}_{D D}\left(\epsilon^{*}\right)$ [and $\left.\mathbf{D}_{D D}\left(\epsilon^{*}\right)\right]$ that fulfills,

$$
\operatorname{Re}\left\{u_{\lambda}^{\dagger} \mathbf{D}_{D D}\left(\epsilon^{*}\right) u_{\lambda}\right\}=0
$$

and

$$
\left.\frac{\partial}{\partial \epsilon} \operatorname{Re}\left\{u_{\lambda}^{\dagger} \mathbf{D}_{D D}(\epsilon) u_{\lambda}\right\}\right|_{\epsilon=\epsilon^{*}}>0
$$

for some energy, $\epsilon^{*}$, corresponding to a peak in DOS.

An illustration of these two conditions is given in Fig. 13. In practice, the modes are found from the number of positive eigenvalues of $\mathbf{D}_{D D}$ evaluated at each point of our energy grid. If this number increases between two successive energies, $\epsilon$ and $\epsilon+\Delta \epsilon$, the eigenmodes at these two energies are matched up. The eigenmode corresponding to the eigenvalue that changes sign is then identified as a mode of the open system. *mads.engelund@nanotech.dtu.dk

${ }^{1}$ G. Cuniberti, G. Fagas, and K. Richter, Introducing Molecular Electronics (Springer, New York, 2005).

${ }^{2}$ G. Schulze et al., Phys. Rev. Lett. 100, 136801 (2008).

${ }^{3}$ Y. Teramae, K. Horiguchi, S. Hashimoto, M. Tsutsui, S. Kurokawa, and A. Sakai, Appl. Phys. Lett. 93, 083121 (2008).

${ }^{4}$ Z. F. Huang, F. Chen, R. D’Agosta, P. A. Bennett, M. Di Ventra, and N. J. Tao, Nat. Nanotechnol. 2, 698 (2007).

${ }^{5}$ M. Galperin, A. Nitzan, and M. A. Ratner, Phys. Rev. B 75, 155312 (2007).

${ }^{6}$ D. A. Ryndyk, P. D'Amico, G. Cuniberti, and K. Richter, Phys. Rev. B 78, 085409 (2008).

${ }^{7}$ M. Tsutsui, M. Taniguchi, and T. Kawai, Nano Lett. 8, 3293 (2008).

${ }^{8}$ Z. Ioffe, T. Shamai, A. Ophir, G. Noy, I. Yutsis, K. Kfir, O. Cheshnovsky, and Y. Selzer, Nat. Nanotechnol. 3, 727 (2008).

${ }^{9}$ G. Rubio-Bollinger, S. R. Bahn, N. Agraït, K. W. Jacobsen, and S. Vieira, Phys. Rev. Lett. 87, 026101 (2001).

${ }^{10}$ V. Rodrigues and D. Ugarte, Phys. Rev. B 63, 073405 (2001).

${ }^{11}$ N. Agraït, C. Untiedt, G. Rubio-Bollinger, and S. Vieira, Chem. Phys. 281, 231 (2002)
${ }^{12}$ N. Agraït, C. Untiedt, G. Rubio-Bollinger, and S. Vieira, Phys. Rev. Lett. 88, 216803 (2002).

${ }^{13}$ S. B. Legoas, D. S. Galvao, V. Rodrigues, and D. Ugarte, Phys. Rev. Lett. 88, 076105 (2002).

${ }^{14}$ N. Agraït, A. L. Yeyati, and J. M. van Ruitenbeek, Phys. Rep. 377, 81 (2003).

${ }^{15}$ L. G. C. Rego, A. R. Rocha, V. Rodrigues, and D. Ugarte, Phys. Rev. B 67, 045412 (2003).

${ }^{16}$ P. Z. Coura, S. B. Legoas, A. S. Moreira, F. Sato, V. Rodrigues, S. O. Dantas, D. Ugarte, and D. S. Galvao, Nano Lett. 4, 1187 (2004).

${ }^{17}$ J. Bettini, V. Rodrigues, J. C. Gonzalez, and D. Ugarte, Appl. Phys. A: Mater. Sci. Process. 81, 1513 (2005).

${ }^{18}$ M. Lagos, V. Rodrigues, and D. Ugarte, J. Electron Spectrosc. Relat. Phenom. 156-158, 20 (2007).

${ }^{19}$ A. Hasmy, L. Rincón, R. Hernández, V. Mujica, M. Márquez, and C. González, Phys. Rev. B 78, 115409 (2008).

${ }^{20}$ T. Kizuka, Phys. Rev. B 77, 155401 (2008).

${ }^{21}$ A. Thiess, Y. Mokrousov, S. Blugel, and S. Heinze, Nano Lett. 8, 2144 (2008).

${ }^{22}$ M. Tsutsui, K. Shoji, M. Taniguchi, and T. Kawai, Nano Lett. 8, 
345 (2008).

${ }^{23}$ T. N. Todorov, Philos. Mag. B 77, 965 (1998).

${ }^{24}$ S. R. Bahn and K. W. Jacobsen, Phys. Rev. Lett. 87, 266101 (2001).

${ }^{25}$ E. Z. da Silva, A. J. R. da Silva, and A. Fazzio, Phys. Rev. Lett. 87, 256102 (2001).

${ }^{26}$ Y.-C. Chen, M. Zwolak, and M. Di Ventra, Nano Lett. 3, 1691 (2003).

${ }^{27}$ M. J. Montgomery, J. Heakstra, T. N. Todorov, and A. P. Sutton, J. Phys.: Condens. Matter 15, 731 (2003).

${ }^{28}$ T. Frederiksen, M. Brandbyge, N. Lorente, and A. P. Jauho, Phys. Rev. Lett. 93, 256601 (2004).

${ }^{29}$ J. K. Viljas, J. C. Cuevas, F. Pauly, and M. Hafner, Phys. Rev. B 72, 245415 (2005).

${ }^{30}$ M. Paulsson, T. Frederiksen, and M. Brandbyge, Phys. Rev. B 72, 201101(R) (2005).

${ }^{31}$ T. Frederiksen, M. Paulsson, M. Brandbyge, and A.-P. Jauho, Phys. Rev. B 75, 205413 (2007).

${ }^{32}$ T. Frederiksen, N. Lorente, M. Paulsson, and M. Brandbyge, Phys. Rev. B 75, 235441 (2007).

${ }^{33}$ E. Hobi, A. Fazzio, and A. J. R. da Silva, Phys. Rev. Lett. 100, 056104 (2008).

${ }^{34}$ H. Yasuda and A. Sakai, Phys. Rev. B 56, 1069 (1997).

${ }^{35}$ R. H. M. Smit, C. Untiedt, and J. M. van Ruitenbeek, Nanotechnology 15, S472 (2004).

${ }^{36}$ M. Tsutsui, Y.-k. Taninnouchi, S. Kurokawa, and A. Sakai, Jpn. J. Appl. Phys., Part 1 44, 5188 (2005).
${ }^{37}$ M. Tsutsui, S. Kurokawa, and A. Sakai, Nanotechnology 17, 5334 (2006).

${ }^{38} \mathrm{~N}$. Agraï (private communications).

${ }^{39}$ J. M. Soler, E. Artacho, J. D. Gale, A. García, J. Junquera, P. Ordejón, and D. Sánchez-Portal, J. Phys.: Condens. Matter 14, 2745 (2002).

${ }^{40} \mathrm{G}$. Tréglia and M. C. Desjonquères, J. Phys. (Paris) 46, 987 (1985).

${ }^{41}$ V. Rodrigues and D. Ugarte, Eur. Phys. J. D 16, 395 (2001).

${ }^{42}$ N. Mingo, D. A. Stewart, D. A. Broido, and D. Srivastava, Phys. Rev. B 77, 033418 (2008).

${ }^{43}$ Formally, this equation is derived by inserting identity operators $I \equiv|D\rangle\langle D|+| \alpha\rangle\langle\alpha|$ in Eq. (3) and using the basis $\{|D\rangle,|\alpha\rangle\}$ for the matrix representation.

${ }^{44}$ M. P. L. Sancho, J. M. L. Sancho, and J. Rubio, J. Phys. F: Met. Phys. 14, 1205 (1984).

${ }^{45}$ N. Mingo, Phys. Rev. B 74, 125402 (2006).

${ }^{46}$ J. S. Wang, J. Wang, and J. T. Lu, Eur. Phys. J. B 62, 381 (2008).

${ }^{47}$ D. Sánchez-Portal, E. Artacho, J. Junquera, P. Ordejón, A. García, and J. M. Soler, Phys. Rev. Lett. 83, 3884 (1999).

${ }^{48}$ The $k$-point sampling of Ref. 31 is so sparse that it may in certain instances give unrealistic predictions for the structure.

${ }^{49}$ P. Velez, S. A. Dassie, and E. P. M. Leiva, Chem. Phys. Lett. 460, 261 (2008).

${ }^{50}$ J. Hihath, C. R. Arroyo, G. Rubio-Bollinger, N. J. Tao, and N. Agrait, Nano Lett. 8, 1673 (2008). 